\title{
COEFICIENTE DE DESCARGA EM EMISSORES DE PIVÔ CENTRAL
}

Doi:http://dx.doi.org/10.1590/1809-4430-Eng.Agric.v35n3p419-429/2015

\section{RENATO M. DA SILVA ${ }^{1}$, RUBENS D. COELHO ${ }^{2}$, LUCAS DO A. FARIA ${ }^{3}$, RAFAEL MASCHIO}

\begin{abstract}
RESUMO: Este trabalho foi desenvolvido com o objetivo de determinar, em condições laboratoriais, o coeficiente de descarga $\left(\mathrm{C}_{\mathrm{d}}\right)$ dos emissores fixos tipo "spray" (Senninger, Nelson e Fabrimar) utilizados em equipamentos pivô central no Brasil. Foram avaliados 45 bocais Senninger, 42 bocais Nelson e 36 bocais Fabrimar, com três repetições, totalizando 369 bocais ensaiados. As pressões de funcionamento variaram entre 6 e 30 PSI (41,37 a 206,84 kPa), com intervalo de leitura a cada 2 PSI $(13,79 \mathrm{kPa})$, totalizando 13 pressões analisadas por bocal. Foram ajustadas as curvas do coeficiente de descarga em função do diâmetro de bocal, para quatro pressões de operação: 10; 15; 20 e 30 PSI $(68,95 ; 103,42 ; 137,90$ e 206,84 kPa). Utilizou-se de dois manômetros de precisão (analógico e digital) previamente calibrados em um manômetro de peso morto. As vazões foram obtidas utilizando três medidores eletromagnético-indutivos, calibrados em recipiente volumétrico. $\mathrm{O}$ diâmetro dos bocais foi mensurado atra vés de uma ampliação em projetor de perfil da marca Starrett, modelo HB 400. Os resultados evidenciaram que o coeficiente de descarga varia em função da pressão e do diâmetro do bocal. Nos ensaios conduzidos, obtiveram-se $\mathrm{C}_{\mathrm{ds}}$ compreendidos na faixa de 0,88 a 0,98 para a faixa de emissores analisados.
\end{abstract}

PALAVRAS-CHAVE: irrigação; hidráulica agrícola, pressão de funcionamento, aplicação de bocais.

\section{NOZZLE DISCHARGE COEFFICIENT FOR CENTER-PIVOT SYSTEM}

ABSTRACT: This study aimed to determine the discharge coefficients $\left(\mathrm{C}_{\mathrm{d}}\right)$ of sprinkler fixednozzles (Senninger, Nelson and Fabrimar), used in Brazilian center-pivot equipment, under laboratory conditions. These tests used the pressure range standard existing in the nozzle market (psi). A set of 45 Senninger, 42 Nelson and 36 Fabrimar nozzles were assessed, with three replications, in a total of 369 tested nozzles. Operating pressures ranged from 6 to $30 \mathrm{psi}$ (41.37 to $206.84 \mathrm{kPa})$, with a reading gap of $2 \mathrm{psi}(13.79 \mathrm{kPa})$, totaling 13 measures for nozzle. Discharge coefficient curves were drawn as function of nozzle size and operating pressures, which were 10 , 15, 20, and 30 psi $(68.95,103.42,137.90$ and $206.84 \mathrm{kPa}$ ). Two precision pressure gauges (one analog and one digital) were used; being calibrated against a dead weight gauge. Flow rates were obtained using three electromagnetic induction gauges calibrated with volumetric data. Nozzle diameters were measured using a profile projector Model HB 400 (Starrett, São Paulo, Brazil). The results evidenced that discharge coefficient varies with nozzle diameter and operating pressure. Tests showed $\mathrm{C}_{\mathrm{ds}}$ within the range of 0.88 to 0.98 , for the as sessed nozzles.

KEYWORDS: Irrigation; Agricultural hydraulic; Operating Pressure; Nozzle Application.

\footnotetext{
${ }^{1}$ Tecgo $^{\circ}$, M.Sc. em Irrigação e Drenagem, Departamento de Engenharia de Biossistemas, ESALQ/USP, Piracicaba - SP, Fone: (0xx61) 3612-6466, renato.esalq@usp.br

${ }^{2}$ Eng $^{\circ}$ A grônomo, Prof. Associado, Departamento de Engenharia de Biossistemas, ESALQ/USP, Piracicaba - SP, rdcoelho@usp.br

${ }^{3}$ Eng $^{\circ}$ A grícola, Doutorando em Irrigação e Drenagem, Departamento de Engenharia de Biossistemas, ESALQ/USP, Piracicaba SP, lucasfr@usp.br

${ }^{4}$ Eng $^{\circ}$ A grônomo, Doutorando em Irrigação e Drenagem, Departamento de En genharia de Biossistemas, ESALQ/USP, Piracicaba SP,rafaelmaschio@usp.br
} 


\section{INTRODUÇÃO}

As recentes tecnologias advindas da irrigação exercem papel fundamental para o aumento da produtividade agrícola, sendo um dos principais instrumentos para a modernização da agricultura brasileira. Isso permite incremento de produtividade, garantindo maior qualidade e rentabilidade do produto agrícola, proporcionando redução do custo unitário de produção, de forma a ofertar alimentos com regularidade ao longo do ano.

De acordo com PAULINO et al. (2011), cerca de 4,45 milhões de hectares são irrigados no Brasil, sendo 2,8 milhões irrigados por sistemas pressurizados. Para COELHO (2007), nos últimos anos, o sistema pivô central vem destacando-se dos demais. KIM et al. (2008) e KING et al. (2009) relataram que grandes avanços foram incorporados nesses sistemas, a exemplo da possibilidade de aplicação independentemente de água e de defensivos agrícolas. Para PETERS et al. (2008), diversas vantagens são destacadas no uso do sistema pivô central, e uma delas é a possibilidade da completa automação do sistema, conforme o experimento, no qual descreveram um sistema de auto mação acionado segundo a temperatura da superfície foliar dos cultivos. A automação desejada e implementada, segundo QUEIROZ et al. (2008), deve ser aquela que considera a informação de sensores no solo, na planta ou na atmosfera, para o acionamento do sistema de irrigação, e não um calendário programável.

A mais recente tecnologia incorporada no sistema pivô central é a aplicação de taxa variável. Para DUKES \& PERRY (2006), o sistema de irrigação por aspersão pivô central adapta-se bem à irrigação de taxa variável se alterados com controle individual dos aspersores.

O dimensionamento da linha lateral do sistema de irrigação pivô central é uma das etapas mais complexas e importantes do projeto. A configuração ou a escolha dos bocais dos emissores constitui uma das principais etapas deste processo, dadas as características particulares de aplicação de água deste sistema. Como no anel externo do pivô a velocidade de deslocamento das torres aumenta, mantendo constante a velocidade angular, o tempo de aplicação diminui nesta área e, para que seja mantida a mesma lâmina aplicada ao longo de toda a área, deve-se aumentar a taxa de aplicação neste anel. Desta forma, é necessário aumentar o diâmetro dos bocais ao longo do equipamento no campo, que, por sua vez, irá mudar o coeficiente de descarga, em função do aumento de vazão e da alteração na geometria do bocal.

As partículas fluidas aproximam-se do orifício descrevendo trajetórias convergentes. Isto obriga o jato formado a sofrer uma contração em uma seção transversal, ocorrendo um valor mínimo de área na seção em uma posição em que os filetes são paralelos, denominada seção contraída. A razão entre a área do jato na seção contraída e a área nominal do orifício define o denominado Coeficiente de Contração [eq.(1)]:

$$
C_{c}=\frac{A_{c}}{A}
$$

em que,

$C_{c}-$ coeficiente de contração, adimensional;

$A_{c} \quad$ - área contraída, $\mathrm{mm}^{2}, \mathrm{e}$

$A \quad$ - área real, $\mathrm{mm}^{2}$.

Devido à existência de perdas de carga localizada (energia hidráulica) durante a passagem da água pelo orifício, sua velocidade real na seção de saída do bocal é ligeiramente menor que a velocidade teórica [eq.(2)], assim como a seção contraída do jato de água é menor do que a seção do bocal.

$$
V_{t}=\sqrt{2 g H}
$$


em que,

$$
\begin{aligned}
& V_{t}-\text { velocidade teórica, } \mathrm{m} \mathrm{s}^{-1} \\
& g-\text { gravidade, } \mathrm{m} \mathrm{s}^{-2}, \mathrm{e} \\
& H \quad-\text { pressão de operação, m.c.a. }
\end{aligned}
$$

A relação entre a velocidade real e a teórica denomina-se coeficiente de velocidade [eq.(3)]:

$$
C_{v}=\frac{V}{V_{t}}
$$

em que,

$C_{v}-$ coeficiente de velocidade, adimensional, e

$V \quad-$ velocidade real, $\mathrm{m} \mathrm{s}^{-2}$.

Ao produto dos coeficientes de contração $\left(\mathrm{C}_{\mathrm{c}}\right)$ e de velocidade $\left(\mathrm{C}_{\mathrm{v}}\right)$ define-se o coeficiente de descarga [eq.(4)]:

$$
C_{d}=C_{c} \cdot C_{v}
$$

em que,

$C_{d} \quad-$ coeficiente de descarga, adimensional.

O objetivo deste trabalho foi determinar os coeficientes de descarga dos emissores comerciais (Senninger, Nelson e Fabrimar), utilizados em sistema de irrigação pivô central, visando a desenvolver equações de regressão para cálculo do $\mathrm{C}_{\mathrm{d}}$, de acordo com o diâmetro do bocal e a pressão de serviço dos emissores.

\section{MATERIAL E MÉTODOS}

O presente estudo foi conduzido em ambiente fechado, no Laboratório de Irrigação do Instituto Nacional de Ciência e Tecnologia em Engenharia da Irrigação (INCTEI), do Departamento de Engenharia de Biossistemas da Escola Superior de Agricultura "Luiz de Queiroz" (ESALQUSP), Piracicaba-SP. A bancada de testes e seus elementos estão esquematizados na Figura 1. Por motivo de segurança experimental, as pressões de ensaio foram monitoradas por dois manômetros simultaneamente: digital e analógico. Este procedimento permite monitorar, com maior segurança, as pressões de ensaio, evitando algum descontrole por tensão elétrica de bateria e pelo amortecimento eletrônico do sistema digital. Os mesmos foram instalados seguindo as normas da Associação Brasileira de Normas Técnicas ABNT (1991), com a tomada de pressão conectada em um ponto localizado aproximadamente a $0,2 \mathrm{~m}$ abaixo do bocal, com o manômetro situado no mesmo plano horizontal (nível) do bocal. 


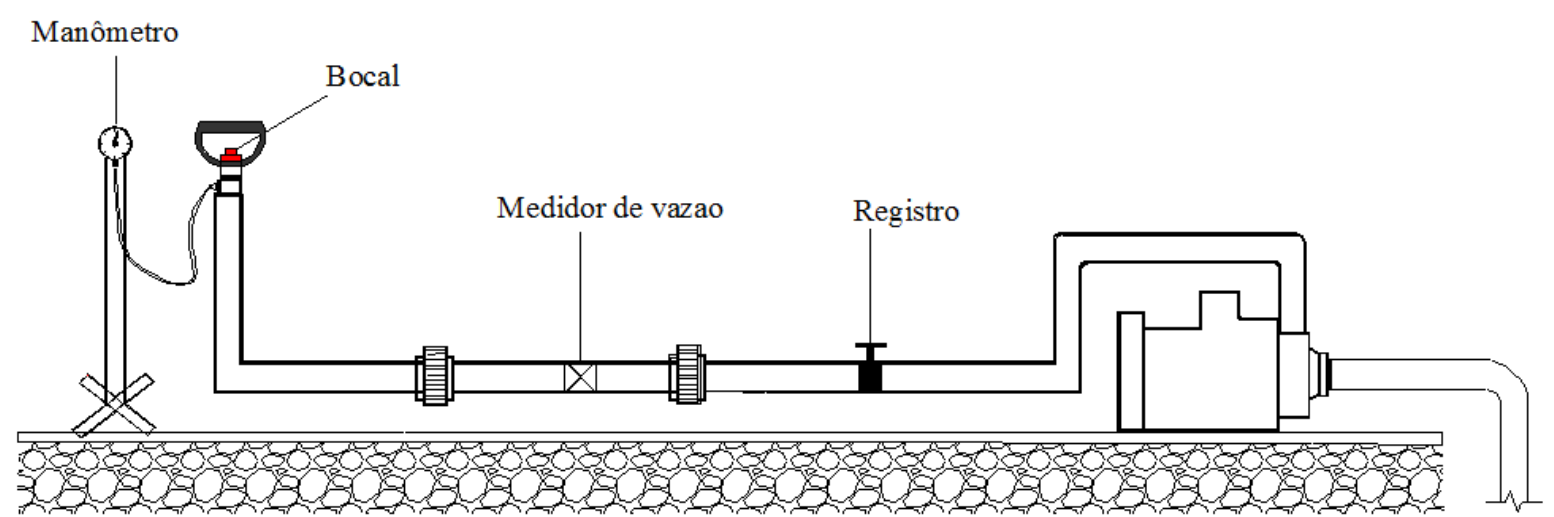

FIGURA 1. Esquema da instalação utilizada na determinação do coeficiente de descarga.

Experimental installation layout for nozzle discharge coefficient de termination.

Devido ao procedimento hidráulico de avaliação (pressões variando de 6 a 30 PSI), utilizou-se de três medidores de vazão eletromagnéticos indutivos (diferentes faixas de vazão), uma vez que a ampla faixa de vazão compreendida entre a menor e a maior vazão do bocal não possibilita sua determinação, utilizando-se apenas de um medidor, em função das características específicas dos medidores magnéticos. Para maior exatidão, foram efetuadas calibrações a cada dois dias de trabalho nos medidores de vazão, utilizando um tanque volumétrico de medição, com capacidade de 1.200 litros, provido de régua com escala graduada.

A seção de escoamento dos bocais foi determinada por meio de um projetor de perfil óptico, marca Starrett, modelo HB 400. Nas Tabelas 1, 2 e 3, são apresentadas as variáveis diâmetro interno e número de identificação dos bocais (NIB), que representam cada bocal de determinado fabricante.

TABELA 1. Características dos bocais Senninger utilizados no ensaio, número de identificação do Bocal (NIB), diâmetro interno (DI) (mm). Characteristics of Senninger nozzles applied in the tests, nozzle identification number (NIB), inner diameter (DI) (mm).

\begin{tabular}{rccccc}
\hline NIB & DI & NIB & DI & NIB & DI \\
\hline 4,0 & 1,599 & 11,5 & 4,595 & 19,0 & 7,595 \\
4,5 & 1,834 & 12,0 & 4,800 & 19,5 & 7,793 \\
5,0 & 2,006 & 12,5 & 4,992 & 20,0 & 8,002 \\
5,5 & 2,229 & 13,0 & 5,181 & 20,5 & 8,184 \\
6,0 & 2,396 & 13,5 & 5,385 & 21,0 & 8,405 \\
6,5 & 2,610 & 14,0 & 5,564 & 21,5 & 8,608 \\
7,0 & 2,799 & 14,5 & 5,792 & 22,0 & 8,785 \\
7,5 & 3,004 & 15,0 & 5,997 & 22,5 & 8,979 \\
8,0 & 3,203 & 15,5 & 6,192 & 23,0 & 9,182 \\
8,5 & 3,390 & 16,0 & 6,403 & 23,5 & 9,388 \\
9,0 & 3,582 & 16,5 & 6,570 & 24,0 & 9,604 \\
9,5 & 3,805 & 17,0 & 6,812 & 24,5 & 9,748 \\
10,0 & 3,987 & 17,5 & 6,986 & 25,0 & 9,966 \\
10,5 & 4,196 & 18,0 & 7,206 & 25,5 & 10,137 \\
11,0 & 4,377 & 18,5 & 7,393 & 26,0 & 10,350 \\
\hline
\end{tabular}


TABELA 2. Características dos bocais Nelson utilizados no ensaio, número de identificação do bocal (NIB), diâmetro interno (DI) (mm). Characteristics of Nelson nozzles applied in the tests, nozzle identification numbe $\mathrm{r}$ (NIB), inner diameter (DI) (mm).

\begin{tabular}{cccccc}
\hline NIB & DI & NIB & DI & NIB & DI \\
\hline 9 & 1,831 & 23 & 4,613 & 37 & 7,410 \\
10 & 2,026 & 24 & 4,915 & 38 & 7,639 \\
11 & 2,213 & 25 & 5,008 & 39 & 7,829 \\
12 & 2,445 & 26 & 5,173 & 40 & 7,977 \\
13 & 2,662 & 27 & 5,398 & 41 & 8,166 \\
14 & 2,810 & 28 & 5,590 & 42 & 8,427 \\
15 & 3,036 & 29 & 5,768 & 43 & 8,621 \\
16 & 3,274 & 30 & 6,042 & 44 & 8,814 \\
17 & 3,402 & 31 & 6,249 & 45 & 9,008 \\
18 & 3,611 & 32 & 6,435 & 46 & 9,197 \\
19 & 3,793 & 33 & 6,617 & 47 & 9,402 \\
20 & 3,997 & 34 & 6,808 & 48 & 9,577 \\
21 & 4,200 & 35 & 7,017 & 49 & 9,797 \\
22 & 4,399 & 36 & 7,252 & 50 & 9,977 \\
\hline
\end{tabular}

TABELA 3. Características dos bocais Fabrimar utilizados no ensaio, número de identificação do bocal (NIB), diâmetro interno (DI) (mm). Characteristics of Fabrimar nozzles applied in the tests, nozzle identification number (NIB), inner diameter (DI) (mm).

\begin{tabular}{cccccc}
\hline NIB & DI & NIB & DI & NIB & DI \\
\hline 1,8 & 1,786 & 4,2 & 4,128 & 6,6 & 6,521 \\
2,0 & 1,950 & 4,4 & 4,351 & 6,8 & 6,750 \\
2,2 & 2,156 & 4,6 & 4,527 & 7,0 & 6,952 \\
2,4 & 2,349 & 4,8 & 4,704 & 7,2 & 7,125 \\
2,6 & 2,595 & 5,0 & 4,980 & 7,4 & 7,328 \\
2,8 & 2,748 & 5,2 & 5,140 & 7,6 & 7,526 \\
3,0 & 2,982 & 5,4 & 5,341 & 7,8 & 7,769 \\
3,2 & 3,147 & 5,6 & 5,561 & 8,0 & 7,934 \\
3,4 & 3,352 & 5,8 & 5,732 & 8,4 & 8,309 \\
3,6 & 3,541 & 6,0 & 5,935 & 8,8 & 8,693 \\
3,8 & 3,751 & 6,2 & 6,134 & 9,2 & 9,109 \\
4,0 & 3,940 & 6,4 & 6,336 & 9,6 & 9,516 \\
\hline
\end{tabular}

Na Figura 2, são ilustrados os perfis de convergência do bocal menor, bocal médio e bocal maior observados experimentalmente, mostrando a variação do ângulo de convergência, com relação aos diâmetros de entrada e saída, conforme a variação do diâmetro do bocal. 


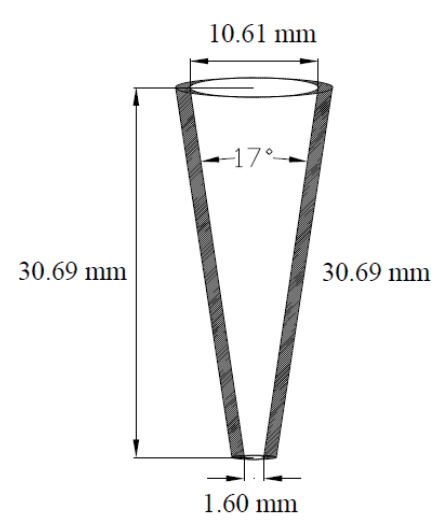

A

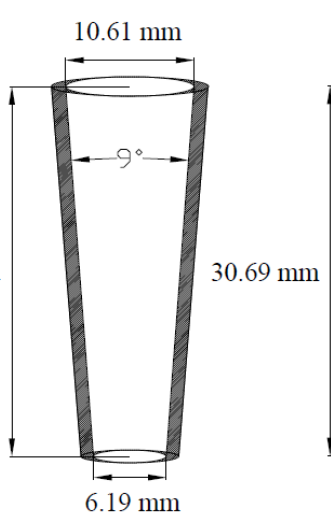

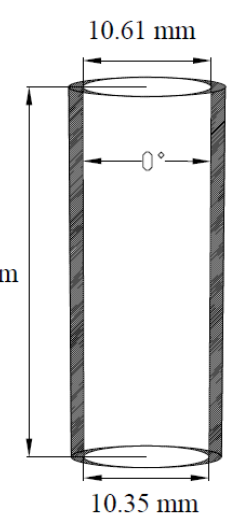

C

FIGURA 2. Ângulo de convergência dos bocais com diâmetros menor, médio e maior observados no estudo. Bocais: (A) Menor, (B) Médio e (C) Maior. Nozzle conve rgence angle of the smallest, medium and largest diameter studied in this experiment. Nozzles: (A) Small, (B) Medium and (C) Large.

\section{Coeficiente de contração}

Com o intuito de observar a ação direta do coeficiente de contração $\left(\mathrm{C}_{\mathrm{c}}\right)$ sobre o coeficiente de descarga $\left(\mathrm{C}_{\mathrm{d}}\right)$, avaliou-se a ocorrência de contração do jato de água na seção do orifício do bocal.

Devido à inércia, o fluxo d'água não pode mudar de direção de forma brusca ao se aproximar da saída, obrigando o jato a se contrair um pouco além da borda interna da abertura, a qual é denominada contração de jato (TEIXEIRA et al., 2007).

A partir de imagens fotográficas, foi avaliada a ocorrência de contração. Utilizando o software AutoCAD 2006, foi determinado o diâmetro do jato d'água, comparando-o com o diâmetro do bocal, determinado com o projetor de perfil óptico.

Foram avaliados os bocais $1,8(1,786 \mathrm{~mm}) ; 6,0(5,935 \mathrm{~mm})$ e $9,6(9,516 \mathrm{~mm})$ fabricados pela Fabrimar, sendo os mesmos submetidos às pressões de $10 ; 15 ; 20$ e 30 PSI.

\section{Coeficiente de descarga}

Os coeficientes de descarga $\left(\mathrm{C}_{\mathrm{d}}\right)$ foram calculados por meio da [eq. (5)], chamada de lei dos orifícios, isolando o $\mathrm{C}_{\mathrm{d}}$ e considerando o diâmetro do orifício para a definição da área.

$$
Q=C d \cdot A \cdot \sqrt{2 g H}
$$

em que,

$Q$ - Vazão que passa pelo bocal, $\mathrm{m}^{3} \mathrm{~s}^{-1}$;

$C_{d}$ - Coeficiente de descarga, adimensional;

$A$ - Área do bocal, $\mathrm{m}^{2}$;

$g$ _ Aceleração da gravidade, $\mathrm{m} \mathrm{s}^{-2}, \mathrm{e}$

$H$ - Pressão de serviço, m.c.a. 


\section{RESULTADOS E DISCUSSÃO}

\section{Coeficiente de descarga}

Na Figura 3, encontram-se as curvas dos coeficientes de descarga $\left(C_{d}\right)$ dos emissores observadas experimentalmente, em função do diâmetro de bocal para quatro pressões de funcionamento $(10 ; 15 ; 20$ e 30 PSI), permitindo observar efeitos da pressão e do diâmetro do bocal no valor do coeficiente de descarga. Pressupõe-se, também, que o efeito quadrático observado nas curvas dos $\mathrm{C}_{\mathrm{ds}}$ ocorre em função do ângulo de convergência que varia com o diâmetro do bocal, reduzindo em ordem crescente o orifício (Figura 2). Os valores obtidos de coeficiente de descarga variaram entre 0,88 a 0,98 . Valores semelhantes foram apresentados por RAPOSO (1979), afirmando que, em sistemas de irrigação por aspersão, o valor de $C_{d}$ não é constante, variando com os valores de diâmetro e pressão de funcionamento, e permanece entre 0,85 e 0,99 . O mesmo autor apresenta um valor médio de $\mathrm{C}_{\mathrm{d}}$ de 0,95 . No entanto, no presente trabalho, os valores médios de $\mathrm{C}_{\mathrm{d}}$ variaram com a pressão de funcionamento e com o fabricante do bocal. Os coeficientes de descarga médios $\left(\mathrm{C}_{\mathrm{dm}}\right)$ apresentados na Tabela 4 foram ajustados para cada conjunto de fabricantes (Senninger, Nelson e Fabrimar) por meio de média aritmética entre os valores de coeficiente de descarga unitária do bocal.

TABELA 4. Valores médios dos coeficientes de descarga em fundação da pressão (PSI) para os fabricantes avaliados. Nozzle discharge coefficient mean values according to pressure (psi) for each evaluated trademark.

\begin{tabular}{cccc}
\hline \multirow{2}{*}{$\begin{array}{c}\text { Pressão } \\
(\mathrm{PSI})\end{array}$} & \multicolumn{3}{c}{ Coeficiente de descarga médio } \\
\cline { 2 - 4 } & Senninger & Nelson & Fabrimar \\
\hline 10 & 0,933 & 0,921 & 0,929 \\
15 & 0,943 & 0,932 & 0,938 \\
20 & 0,950 & 0,940 & 0,947 \\
30 & 0,958 & 0,947 & 0,956 \\
\hline
\end{tabular}

Nas Tabelas 5, 6 e 7, encontram-se as equações ajustadas em função da pressão de funcionamento e do diâmetro do bocal. Observa-se um efeito quadrático significativo $(p<0,01)$ para a variável diâmetro em todas as pressões de funcionamento avaliadas. $O$ coeficiente de determinação das equações polinomiais variou com o fabricante e com a pressão de funcionamento do bocal, na faixa de 0,719 a 0,932 (Tabelas 5, 6 e 7).
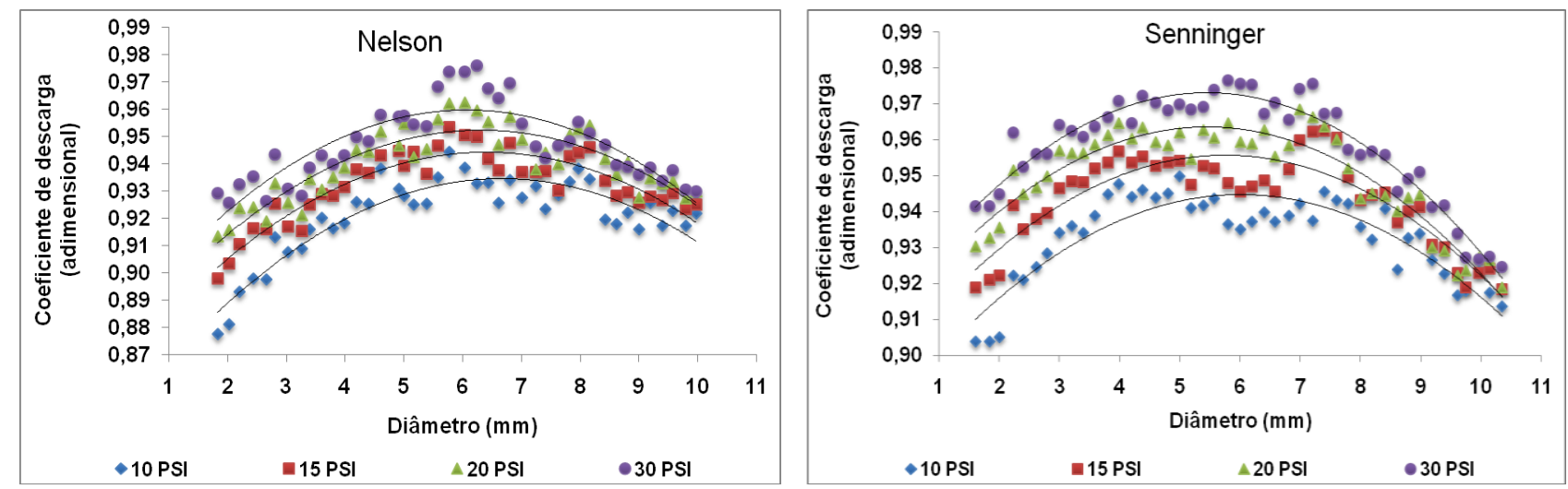


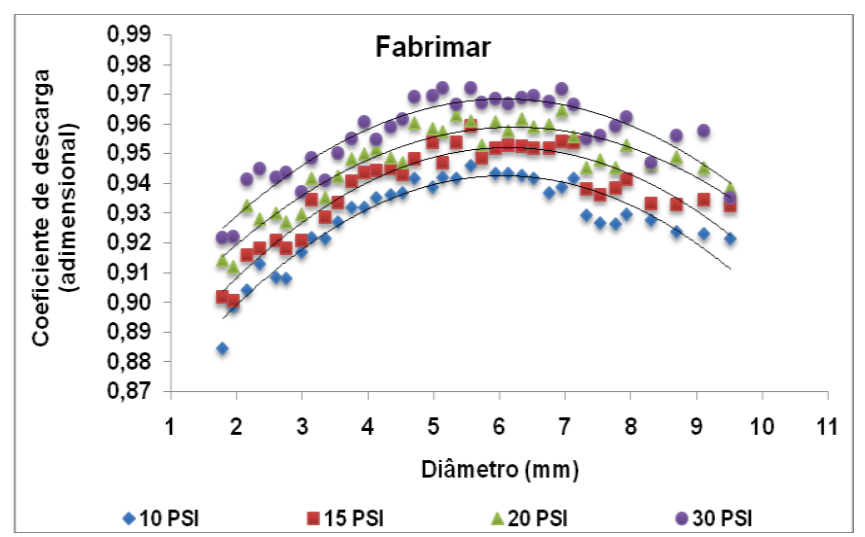

FIGURA 3. Curvas dos coeficientes de descarga dos bocais Senninger, Nelson e Fabrimar em função do diâmetro do bocal e da pressão de funcionamento. Nozzle discharge coefficient curves for Senninger, Nelson and Fabrimar nozzles in vie w of the nozzle diameter and operating pressure.

TABELA 5. Equações dos coeficientes de descarga dos bocais Senninger em função da pressão e do diâmetro do bocal ( $\mathrm{mm})$. Nozzle discharge coefficient equations for Senninger nozzles according to nozzle diameter $(\mathrm{mm})$.

\begin{tabular}{cccc}
\hline $\begin{array}{c}\text { Pressão } \\
(\mathrm{PSI})\end{array}$ & Equação & $\mathrm{F}$ & $\mathrm{R}^{2}$ \\
\hline 10 & $\mathrm{C}_{\mathrm{d}}=-0,0017978 \mathrm{D}^{2}+0,0215875 \mathrm{D}+0,8799767$ & $* *$ & 0,7987 \\
15 & $\mathrm{C}_{\mathrm{d}}=-0,0018565 \mathrm{D}^{2}+0,0213390 \mathrm{D}+0,8944407$ & $* *$ & 0,8049 \\
20 & $\mathrm{C}_{\mathrm{d}}=-0,0019732 \mathrm{D}^{2}+0,0215027 \mathrm{D}+0,9049165$ & $* *$ & 0,8883 \\
30 & $\mathrm{C}_{\mathrm{d}}=-0,0021499 \mathrm{D}^{2}+0,0234275 \mathrm{D}+0,9092209$ & $* *$ & 0,9329 \\
\hline
\end{tabular}

** Signif icativo a $1 \%$ de probabilidade

TABELA 6. Equações dos coeficientes de descarga dos bocais Nelson em função da pressão e do diâmetro do bocal ( $\mathrm{mm})$. Nozzle discharge coefficient equations for Nelson nozzles according to nozzle diameter $(\mathrm{mm})$.

\begin{tabular}{cccc}
\hline $\begin{array}{c}\text { Pressão } \\
\text { (PSI) }\end{array}$ & Equação & $\mathrm{F}$ & $\mathrm{R}^{2}$ \\
\hline 10 & $\mathrm{C}_{\mathrm{d}}=-0,0020883 \mathrm{D}^{2}+0,0278723 \mathrm{D}+0,8415777$ & $* *$ & 0,8410 \\
15 & $\mathrm{C}_{\mathrm{d}}=-0,0020194 \mathrm{D}^{2}+0,0258972 \mathrm{D}+0,8613968$ & $* *$ & 0,8463 \\
20 & $\mathrm{C}_{\mathrm{d}}=-0,0020457 \mathrm{D}^{2}+0,0258758 \mathrm{D}+0,8704095$ & $* *$ & 0,7907 \\
30 & $\mathrm{C}_{\mathrm{d}}=-0,0022330 \mathrm{D}^{2}+0,0271460 \mathrm{D}+0,8773646$ & $* *$ & 0,7198 \\
\hline
\end{tabular}

** Significativo a $1 \%$ de probabilidade

TABELA 7. Equações dos coeficientes de descarga dos bocais Fabrimar em função da pressão e do diâmetro do bocal $(\mathrm{mm})$. Nozzle discharge coefficient equations for Fabrimar nozzles according to nozzle diameter $(\mathbf{m m})$.

\begin{tabular}{cccc}
\hline $\begin{array}{c}\text { Pressão } \\
\text { (PSI) }\end{array}$ & Equação & $\mathrm{F}$ & $\mathrm{R}^{2}$ \\
\hline 10 & $\mathrm{C}_{\mathrm{d}}=-0,0026382 \mathrm{D}^{2}+0,0319711 \mathrm{D}+0,8456675$ & $* *$ & 0,9003 \\
15 & $\mathrm{C}_{\mathrm{d}}=-0,0025697 \mathrm{D}^{2}+0,0314725 \mathrm{D}+0,8555731$ & $* *$ & 0,8944 \\
20 & $\mathrm{C}_{\mathrm{d}}=-0,0021887 \mathrm{D}^{2}+0,0272734 \mathrm{D}+0,8738154$ & $* *$ & 0,8738 \\
30 & $\mathrm{C}_{\mathrm{d}}=-0,0023644 \mathrm{D}^{2}+0,0286654 \mathrm{D}+0,8813079$ & $* *$ & 0,8501 \\
\hline ** Significativo a 1\% de probabilidade
\end{tabular}




\section{Coeficiente de contração}

Para ZITTERELL et al. (2009), a perda localizada de carga depende da geometria da peça e da velocidade de escoamento do fluido. Para os bocais avaliados, o corpo do emissor é dimensionado de forma a coincidir o diâmetro de saída com o diâmetro de entrada do bocal. Assim, as peças unem-se dando seguimento ao perfil de convergência dos orifícios (orifício do corpo do emissor e do bocal). Desta forma, a perda de energia do fluido é menor que em bocais com bordas acentuadas. AZEVEDO NETTO et al. (1998) afirmam que não ocorre contração no fluido em escoamento para bocais cônicos convergentes. De modo semelhante, avaliando as imagens fotográficas com o software AutoCAD 2006, não foi possível observar a contração do jato de água na saída do bocal. Ao passar pela aresta do orifício, o jato tende a se expandir e, logo após a saída do bocal, o diâmetro do jato d'água já é ligeiramente superior ao diâmetro do bocal (Tabela 8).

TABELA 8. Diâmetro do jato d'água dos bocais Fabrimar utilizados no ensaio. Water jet diameter of Fabrimar nozzles.

\begin{tabular}{cccccc}
\hline \multirow{2}{*}{ Diâmetro nominal do bocal } & \multirow{2}{*}{ Diâmetro interno do bocal } & \multicolumn{4}{c}{ Diâmetro do jato d'água (mm) } \\
\cline { 3 - 5 }$(\mathrm{mm})$ & $(\mathrm{mm})$ & \multicolumn{4}{c}{ Pressão (PSI) } \\
\cline { 3 - 5 } & 1,786 & 10 & 15 & 20 & 30 \\
\hline 1,8 & 5,935 & 6,297 & 1,807 & 1,788 & 1,833 \\
6,0 & 9,516 & 9,853 & 9,749 & 9,288 & 6,300 \\
9,6 & & &
\end{tabular}

\section{Relação núme ro do bocal e diâmetro real}

$\mathrm{Na}$ Figura 4, são apresentados os diâmetros internos dos bocais observados experimentalmente, em função do número de identificação, para as três marcas estudadas. As equações ajustadas podem ser observadas na Tabela 9. Para todos os fabricantes, as equações apresentaram coeficiente de determinação superior a 0,999 para o modelo linear.

Nas Tabelas 10, 11 e 12, podem ser observados os coeficientes de variação dos diâmetros dos bocais a valiados (Senninger, Nelson e Fabrimar).
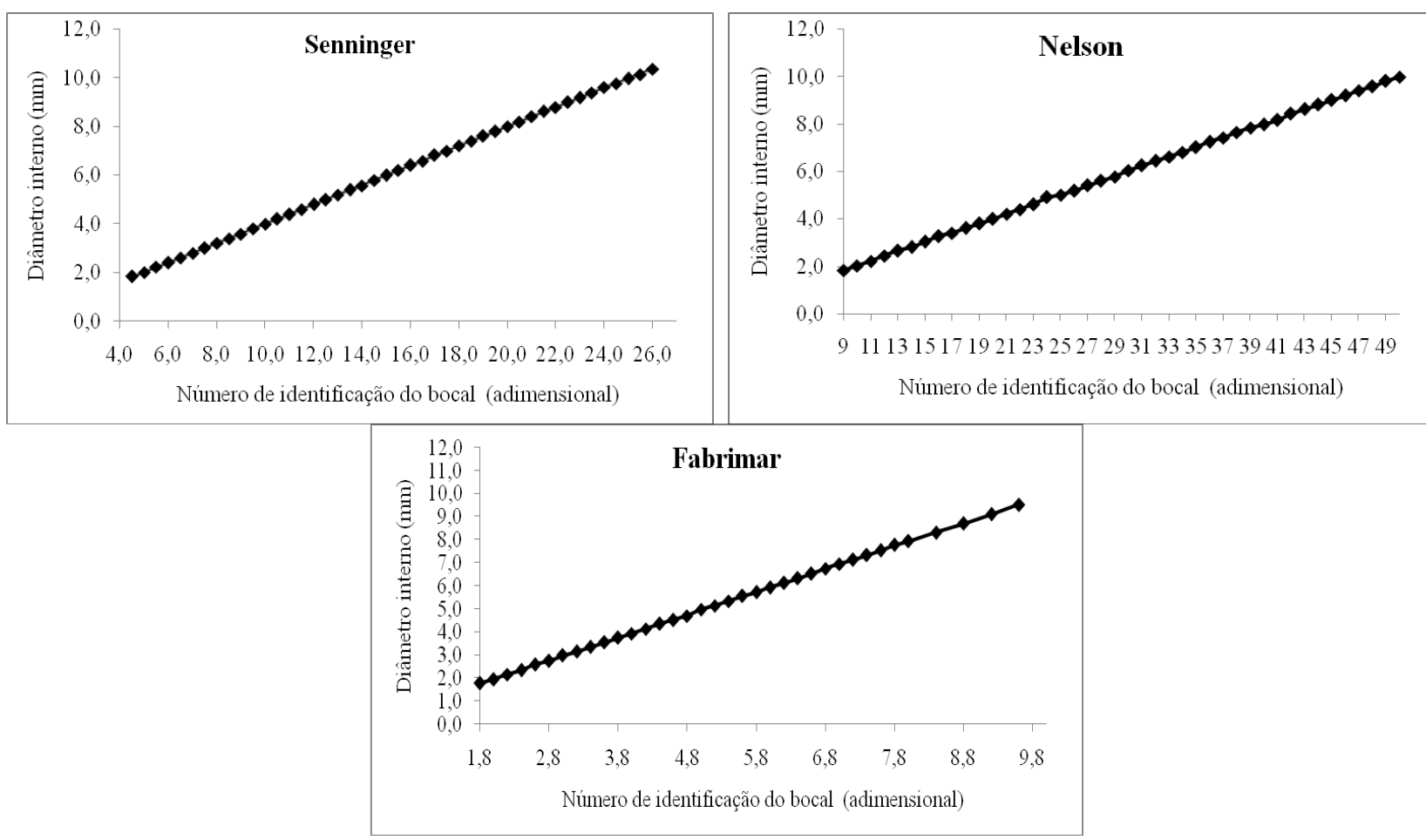

FIGURA 4. Diâmetro interno ( $\mathrm{mm}$ ) em função do número de identificação do bocal (adimensional). Nozzle inner diameter $(\mathrm{mm})$ according to nozzle identification number (dimensionless). 
TABELA 9. Equações do diâmetro interno dos bocais em função do número de identificação (adimensional) para os fabricantes estudados. Nozzle inner diameter equations according to nozzle identification number (dimensionless) for the evaluated trade marks.

\begin{tabular}{ccc}
\hline Marca & Equação do Diâmetro Interno (mm) & $\mathrm{R}^{2}$ \\
\hline Senninger & $\mathrm{D}_{\mathrm{i}}=0,398 \mathrm{NO}+0,014$ & 0,999 \\
Nelson & $\mathrm{D}_{\mathrm{i}}=0,199 \mathrm{NO}+0,037$ & 0,999 \\
Fabrimar & $\mathrm{D}_{\mathrm{i}}=0,993 \mathrm{DN}+0,024$ & 0,999 \\
\hline
\end{tabular}

TABELA 10. Coeficiente de variação do diâmetro dos bocais Senninger utilizados no ensaio, número de identificação dos bocais (NIB), coeficiente de variação $\left(\mathrm{C}_{\mathrm{v}}\right)$. Diameter variation coefficient of Senninger nozzles, nozzle identification number (NIB), coefficient of variation $\left(\mathrm{C}_{\mathrm{v}}\right)$.

\begin{tabular}{cccccccccc}
\hline NIB & $\mathrm{C}_{\mathrm{v}}(\%)$ & NIB & $\mathrm{C}_{\mathrm{v}}(\%)$ & $\mathrm{NIB}$ & $\mathrm{C}_{\mathrm{v}}(\%)$ & $\mathrm{NIB}$ & $\mathrm{C}_{\mathrm{v}}(\%)$ & $\mathrm{NIB}$ & $\mathrm{C}_{\mathrm{v}}(\%)$ \\
\hline 4,0 & 0,982 & 8,5 & 0,213 & 13,0 & 1,014 & 17,5 & 0,433 & 22,0 & 0,662 \\
4,5 & 0,164 & 9,0 & 0,540 & 13,5 & 0,639 & 18,0 & 0,407 & 22,5 & 0,636 \\
5,0 & 0,225 & 9,5 & 0,315 & 14,0 & 0,615 & 18,5 & 0,453 & 23,0 & 0,757 \\
5,5 & 0,202 & 10,0 & 0,151 & 14,5 & 0,635 & 19,0 & 0,759 & 23,5 & 0,659 \\
6,0 & 0,284 & 10,5 & 0,185 & 15,0 & 0,521 & 19,5 & 0,423 & 24,0 & 0,569 \\
6,5 & 0,378 & 11,0 & 0,590 & 15,5 & 0,494 & 20,0 & 0,475 & 24,5 & 0,219 \\
7,0 & 0,095 & 11,5 & 0,712 & 16,0 & 0,750 & 20,5 & 0,420 & 25,0 & 0,112 \\
7,5 & 0,135 & 12,0 & 0,594 & 16,5 & 0,655 & 21,0 & 0,498 & 25,5 & 0,032 \\
8,0 & 0,334 & 12,5 & 0,394 & 17,0 & 0,489 & 21,5 & 0,503 & 26,0 & 0,261 \\
\hline
\end{tabular}

TABELA 11. Coeficiente de variação do diâmetro dos bocais Nelson utilizados no ensaio, número de identificação dos bocais $(\mathrm{NIB})$, coeficiente de variação $\left(\mathrm{C}_{\mathrm{v}}\right)$. Diameter variation coefficient of Nelson nozzles, nozzle identification number (NIB), coefficient of variation $\left(\mathbf{C}_{\mathrm{v}}\right)$.

\begin{tabular}{cccccccccccc}
\hline NIB & $\mathrm{C}_{\mathrm{v}}(\%)$ & NIB & $\mathrm{C}_{\mathrm{v}}(\%)$ & NIB & $\mathrm{C}_{\mathrm{v}}(\%)$ & $\mathrm{NIB}$ & $\mathrm{C}_{\mathrm{v}}(\%)$ & $\mathrm{NIB}$ & $\mathrm{C}_{\mathrm{v}}(\%)$ & $\mathrm{NIB}$ & $\mathrm{C}_{\mathrm{v}}(\%)$ \\
\hline 9 & 0,351 & 16 & 0,341 & 23 & 0,554 & 30 & 1,057 & 37 & 0,339 & 44 & 0,298 \\
10 & 0,747 & 17 & 0,855 & 24 & 1,386 & 31 & 1,674 & 38 & 0,197 & 45 & 0,327 \\
11 & 0,294 & 18 & 0,872 & 25 & 0,366 & 32 & 0,830 & 39 & 0,562 & 46 & 0,358 \\
12 & 0,732 & 19 & 0,448 & 26 & 0,979 & 33 & 0,434 & 40 & 0,115 & 47 & 0,424 \\
13 & 0,413 & 20 & 1,192 & 27 & 0,761 & 34 & 1,748 & 41 & 0,078 & 48 & 0,397 \\
14 & 0,646 & 21 & 0,221 & 28 & 0,514 & 35 & 0,407 & 42 & 0,576 & 49 & 0,325 \\
15 & 0,761 & 22 & 0,208 & 29 & 0,600 & 36 & 0,882 & 43 & 0,984 & 50 & 0,523 \\
\hline
\end{tabular}

TABELA 12. Coeficiente de variação do diâmetro dos bocais Fabrimar utilizados no ensaio, número de identificação dos bocais (NIB), coeficiente de variação $\left(\mathrm{C}_{\mathrm{v}}\right)$. Diameter variation coefficient of Fabrimar nozzles, nozzle identification number (NIB), coefficient of variation $\left(\mathrm{C}_{\mathrm{v}}\right)$.

\begin{tabular}{cccccccccccc}
\hline NIB & $\mathrm{C}_{\mathrm{v}}(\%)$ & NIB & $\mathrm{C}_{\mathrm{v}}(\%)$ & $\mathrm{NIB}$ & $\mathrm{C}_{\mathrm{v}}(\%)$ & $\mathrm{NIB}$ & $\mathrm{C}_{\mathrm{v}}(\%)$ & $\mathrm{NIB}$ & $\mathrm{C}_{\mathrm{v}}(\%)$ & $\mathrm{NIB}$ & $\mathrm{C}_{\mathrm{v}}(\%)$ \\
\hline 1,8 & 0,171 & 3,0 & 0,958 & 4,2 & 0,394 & 5,4 & 0,184 & 6,6 & 1,086 & 7,8 & 0,101 \\
2,0 & 0,157 & 3,2 & 0,814 & 4,4 & 1,082 & 5,6 & 0,371 & 6,8 & 0,034 & 8,0 & 0,131 \\
2,2 & 1,008 & 3,4 & 0,091 & 4,6 & 0,389 & 5,8 & 0,219 & 7,0 & 0,044 & 8,4 & 0,277 \\
2,4 & 2,144 & 3,6 & 0,849 & 4,8 & 0,476 & 6,0 & 0,202 & 7,2 & 0,056 & 8,8 & 0,073 \\
2,6 & 0,456 & 3,8 & 0,429 & 5,0 & 0,471 & 6,2 & 0,114 & 7,4 & 0,116 & 9,2 & 0,203 \\
2,8 & 0,379 & 4,0 & 0,432 & 5,2 & 0,085 & 6,4 & 0,274 & 7,6 & 0,161 & 9,6 & 0,016 \\
\hline
\end{tabular}




\section{CONCLUSÕES}

O coeficiente de descarga dos bocais estudados, utilizados em sistemas de irrigação pivô central, está compreendido entre 0,88 e 0,98 .

O coeficiente de descarga varia em função da pressão de funcionamento e do diâmetro do bocal.Não ocorre contração do jato de água em bocais cônicos convergentes com diâmetro interno de 1,60 até $10,35 \mathrm{~mm}$ e ângulo com até $17^{\circ}$ graus em relação aos diâmetros de entrada e saída do bocal.

\section{AGRADECIMENTOS}

Ao Ministério da Ciência e Tecnologia (MCT), à Coordenação de Aperfeiçoamento de Pessoal de Nível Superior (CAPES), e ao Instituto Nacional de Ciência e Tecnologia em Engenharia da Irrigação (INCTEI), pela disponibilização de equipamentos utilizados no desenvolver do experimento.

\section{REFERÊNCIAS}

ABNT. ASSOCIAÇÃO BRASILEIRA DE NORMAS TÉCNICAS. NBRISO 9261. Equipamentos de irrigação agrícola - Emissores e tubos emissores - Especificação e métodos de ensaio São Paulo, 1991.13p.

COELHO, R.D. Contribuições para a irrigação pressurizada no Brasil. 2007. 192 f. Tese (Livre Docência) - Escola Superior de Agricultura "Luiz de Queiroz", Universidade de São Paulo, Piracicaba, 2007.

AZEVEDO NETTO, J.M.; FERNANDEZ, M.F,; ARAÚJO, R,; ITO, A.F. Manual de hidráulica. 8.ed. São Paulo: Ed gard Blucher, 1998. 670 p.

DUKES, M.D., PERRY, C. Uniformity testing of variable-rate center pivot irrigation control systems. Precision Agriculture, Netherlands, v.7, n.3, p.205-218, 2006.

KIM, Y.; EVANS, R.G.; IVERSEN, W.M. Remote sensing and control of an irrigation system using a distributed wireless sensor network. IEEE, Transactions on Instrumentation and Measurement, New York, v.57, n.7, 2008.

KING, B. A.; WALL, R.W.; KARSKY, T.F. Center-pivot irrigation system for independent sitespecific management of water and chemical application. Applied Engineering in Agriculture, St Joseph, v.25, n.2, p.187-198, 2009.

PAULINO, J.; FOLEGATTI, M.V.; ZOLIN, C.A.; ROMÁN, R.M.S.; JOSÉ, J.V. Situação da agricultura irrigada no brasil de acordo com o censo agropecuário 2006. Irriga, Botucatu, v. 16, n. 2, p. 163-176, 2011.

PETERS, R. T.; EVETT, S. R. Automation of a center pivot using the temperature-time-threshold method of irrigation scheduling. Journal of Irrigation and Drainage Engineering, New York, v.134. n.3, p.286-291, 2008.

QUEIROZ, T.M.; BOTREL, T.A.; FRIZZONE, J.A. Desenvolvimento de software e hardware para irrigação de Precisão usando pivô central. Engenharia Agrícola, Jaboticabal, v.28, n.1, p.44- 54, 2008.

RAPOSO, J.R. A rega por aspersão. Lisboa: Livraria Clássica, 1979. 339 p.

TEIXEIRA, M.B.; MELO, R.F.; COELHO, R.D. Coeficiente de descarga em emis sores de pivô central. Irriga, Botucatu, v.12, n.1, p.123- 134, 2007.

ZITTERELL, D.B.; FRIZZONE, J.A.; RETTORE NETO, O.; ULLMANN, M.N. Perda de carga em microtubos e conectores utilizados em microaspersão. Engenharia Agrícola, Jaboticabal, v.29, n.4, p.591- 604, 2009. 